Aus dem Peter-Paul-Krankenhause zu St. Petersburg.

\title{
Ein Fall von Perivaginitis phlegmonosa dissecans.
}

Von

\author{
Dr. med. Leo von Lingen.
}

Die Perivaginitis phlegmonosa dissecans ist eine Erkrankungsform, die nicht zu den häufigen Erscheinungen gehört, wenigstens ist die Anzahl der in der Literatur publicirten Fälle keine grosse. Dabei fällt die Mehrzahl dieser auf den Antheil russischer Autoren. Erst in jüngster Zeit ist diese Erkrankungsform von deutschen Autoren beschrieben worden, worauf ich später zurückkomme. Die in russischer Sprache erschienenen Arbeiten sind mir sämmtlich im Original zugänglich gewesen und werde ich mir daher erlauben, auf letztere in Kürze einzugehen. --

Die Benennung "Perivaginitis phlegmonosa dissecans" stammt von Marconnet') in Moskau aus dem Jahre 1864, der zwei Fälle einer „besonderen Form der Entzündung der Vagina, die nirgends beschrieben wird" und sich durch Abstossung eines Theiles oder der ganzen Vaginalwand mit Portio vaginalis charakterisirt, beobachtet und unter obiger Benennung veröffentlicht hat.

Seine Fälle bezogen sich auf zwei jugendliche Individuen, welche unter fieberhaften Erscheinungen, Schmerzen im Unterleib und blutigem Ausfluss ohne nachweisbare Ursache erkrankt waren. Aus den Geschlechtstheilen wurde ein Fremdkörper, welcher das Aussehen eines häutigen Sackes hatte und sich als die Vaginalwand selbst (Membrana mucosa und muscularis) mitsammt der Portio Band 34.

1) Moskowsk. mediz. gazeta. 1865. No. 4. p. 33. - Virchow's Archiv. 
596 v. Lingen, Ein Fall von Perivaginitis phlegmonosa dissecans.

vaginalis auswies, ausgestossen. Beide Fälle gingen in Genesung über. Die spontane Ablösung der Port. vag. uteri mit der Vagina befestigt nach Marconnet die Meinung der Zusammengehörigkeit der Portio vaginalis mit der Scheide, aus deren primären Elementen sie sich entwickelt. Die Ursache des ganzen Prozesses sieht der oben genannte Autor in einer Entzündung, welche die Bildung von Eiter in dem Bindegewebe, welches die Vagina umgiebt, veranlasst.

Minkiewitseh ${ }^{1}$ ) in Tiflis beschreibt einen unter schweren Allgemeinerscheinungen zum letalen Ende führenden Fall von Perivaginitis phlegmonosa dissecans. Es handelte sich hier um die Abstossung eines Hohlcylinders, welcher aus der inneren Fläche der Vaginalwand und einem Theil der Port. vag. bestand. Bei der Section, die sich nur auf die Beckenhöhle beschränkte, erwies es sich, dass die vordere Mastdarmwand und die hintere Harnblasenwand zerstört waren. Ein anamnestisches Moment war nicht festzustellen, Verf. ist geneigt den anatomischen Prozess von einer phlegmonösen Entzündung des die angrenzenden Theile umgebenden Bindegewebes herzuleiten, doch giebt er der Vermuthung Raum, dass Thrombose oder Embolie gewisser Gefässe ein aetiologisches Moment abzugeben im Stande wäre. -

Hieran reiht sich ein von Wiegandt2) publicirter Fall an, der den von Marconnet beschriebenen Fällen als Analogon zur Seite gestellt werden kann, indem auch hier ein schlauchförmiges, $7 \mathrm{~cm}$. langes Gebilde, das aus Schleimhaut und Muskelschicht der Scheide besteht, ausgeschieden wurde. Patientin genas unter stärkerer Stenose der Scheide, wurde schwanger, entzog sich aber der weiteren Beobachtung. Wiegandt beschränkt sich auf die Beschreibung der Krankengeschichte, ohne auf die dunkle Seite der Aetiologie einzugehen.

Bei Gelegenheit der Besprechung der dissecirenden Gebärmutterentzündung erwähnt Syromjatnikoff ${ }^{\dot{3}}$ ) einen von Zirkunhenko in Moskau beobachteten Fall, der sich auf eine 35 jährige fieberhaft erkrankte Patientin bezieht. Das ausgestossene $10 \mathrm{~cm}$. lange, sackförmige Stück stellte den ganzen Ausguss der Scheide nebst Port. vagin. dar. Die microscopische Untersuchung zeigte,

1) Virchow's Archiv. Bd. 41.

2) Petersburger med. Wochenschr. 1876. No. 37.

3) Wratsch. 1880. No. 29 u. 30. - Dieses Archiv. 1881. Bd. XVIII. Heft 1. 
dass die Wand des Sackes aus Schleimhaut und submucösem Bindegewebe, das sich als amorphe Masse praesentirte und seine fasrige Structur grösstentheils eingebüsst hatte, mit Beimengung einer geringen Anzahl glatter Muskelfasern, bestand. Bei dieser Untersuchung ist zum ersten Mal dem Verhalten der Gefässe Aufmerksamkeit geschenkt worden: die Gefässe waren zum Theil mit zersetzten Thromben gefüllt, zum Theil waren sie leer.

Syromjatnikoff's Arbeit hat die Metritis dissecans zum Gegenstand, während er den von. Zirkunhenko beobachteten Fall als Analogon erwähnt. In der That lässt sich nicht leugnen, dass der sich im Uterus und Vaginalwand abspielende Prozess in anatomischer Beziehung grosse Aehnlichkeit besitzt, wie wir weiter unten sehen werden.

Tschernischeff ${ }^{1}$ ) berichtet über zwei Fälle aus seiner eignen Praxis, von denen, sich der eine an einen Typhus abdominalis, der andere an eine acute fieberhafte nicht diagnosticirte Krankheit anschloss. In beiden Fällen wurden in mehr weniger ausgedehntem Maasse Stücke aus der Scheidenwand, dem Scheidengewölbe und der Port. vagin. ausgestossen. Die beiden Kranken genasen beide. Im ersten der beiden Fälle fand sich nach Ausstossung des necrotischen Stückes eine Rectovaginalfistel. Letzteres zeigte unter dom Microscop theils noch erkennbares, theils in körnigem Zerfall begriffenes Muskelgewebe, Untergang des Epithels, Infiltration des submucösen Gewebes; die Blutgefässe waren theils leer, theils mit rothen und weissen Blutkörperchen angefüllt. Die Aetiologie der Periraginitis phlegmonosa dissecans stellt Tschernischeff als völlig dunkel hin.

In einem anderen von Dobbert ${ }^{2}$ ) publicirten Fall spielt gleichfalls der Typhus abdom. eine aetiologische Rolle. Es wurde ein $8 \mathrm{~cm}$. langes und mehrere Centimeter breites Gewebsstück aus der Scheide ausgestossen. Bei der Section fand sich neben den Erscheinungen des fast abgelaufenen Unterleibstyphus im hinteren Scheidengewölbe eine Perforationsöffnung von fast $2 \mathrm{~cm}$. im Durchmesser, durch welche die Scheide mit der Bauchhöhle communicirte. Als Analogon führt Dobbert zwei im Peter-PaulHospital beobachtete Fälle von Metritis dissecans im Anschluss an Endometritis puerperalis an.

1) Medizinsk. Westn. 1880. No. 50 u. 51. - Centralbl. f. Gynäkol. 1881. N.o. 5 .

2) Petersburger med. Wochenschr. 1890. No. 23. 
598 v. Lingen, Ein Fall von Perivaginitis phlegmonosa dissecans.

Chanutin ${ }^{1}$ ) führt einen Fall an, der eine 35 jährige Patientin mit exacerbirender Nephritis,Pneumonia catarrh. sin. inf. und Enteritis betraf. Hier löste sich am zweiten Tage des Aufenthaltes im Krankenhause ein $10 \mathrm{~cm}$. langes, aus Port. vag. und vornehmlich von der rechten Seite der Scheidenwand herrührendes necrotisches Stück ab. Daher will Chanutin seinen Fall als Paravaginitis phlegmonosa dissecans partialis bezeichnen. Die Kranke wurde nach $3 \frac{1}{2}$ Monaten geheilt, aber mit einer Verengung des Scheidenlumens, entlassen.

Weber ${ }^{2}$ ) publicirt dje Krankengeschichte einer 38jährigen Patientin, welche unter den Erscheinungen einer fieberhaften Erkrankung in Behandlung kam und unter recht heftigen Blutungen ein cylinderförmiges necrotisches Gewebsstück aus der Scheide ausstiess, das, wie die mikroskopische Untersuchung bestätigte, aus Gewebselementen der Scheidenwandung bestand. Patientin genas alsbald, behielt aber eine Scheidenstenose zurück. Weber beschuldigt in seinem Fall den Streptococus pyogenes, der in dem jauchigen Secret gefunden wurde, als Erreger des Entzündungsprozesses. Die eitrige Entzündung im perivaginalen Gewebe komme entweder dadurch zu Stande, dass vorher stattgebabte traumatische Einflüsse die Integrität der schützenden Epitheldecke der Vagina aufgehoben und damit der Invasion von Microorganismen Gelegenheit gegeben wäre, in die Tiefe einzudringen, oder dass die Gewebselemente durch infectiöse Einflüsse in ihrer vitalen Energie herabgesetzt wärden und leichter dem Eindringen virulenter Microorganismen unterliegen.

Soweit die Litteratur, soweit sich diese auf Arbeiten russischer Autoren bezieht.

In Deutschland scheint Bröse ${ }^{3}$ ) zuerst einen Fall von Perivaginitis dissecans beobachtet zu haben. Bröse demonstrirte ein $13^{1 / 2} \mathrm{~cm}$ langes und $4^{1} / 2-6 \mathrm{~cm}$ breites Stück des Scheidenrobres, das eine Kranke nach kritischem Abfall einer croupösen Pneumonie ausgestossen hatte. Die Kranke genas.

In den deutschen Lehrbüchern der Gynäkologie und pathologischen Anatomie findet obige Erkrankung entweder gar keine

1) Bolnitschn. gaset. 1891. No. 32-34.

2) Shurnal akusch. 1896. No. 12.

3) Zeitschr. f. Geb. u. Gyn. Bd. XXIV. 1892. - Verh. d. Ges. f. Geb. u. Gyn. zu Berlin. S. 362 . 
Erwähnung (Thomas, Ziegler, Langerhans) oder es wird ohne eingehende Schilderung mit wenigen Worten als auf eine seltene Erkrankungsform hingewiesen (Fritsch, Pozzi, Schroeder-Hofmeier). Nur J. Veit') widmet mit Berufung auf die Mehrzahl der oben citirten Arbeiten einige Worte der Betrachtung der Perivaginitis und stellt dieselbe als eine sehr seltene Affection hin.

Der Vollständigkeit halber führe ich noch Bizzozero') an, der bei einer 38 jährigen an catarrhalischer Bronchitis fieberhaft erkrankten Frau ein sackförmiges Stück Vaginalwand und Port. vag. sich abstossen sah. Pat. machte eine Perimetritis durch und genas unter bedeutender Verengerung des Scheidenlumens.

Nicht zugänglich im Original und in der folgenden statistischen Zusammenstellung wegen widersprechender Widergabe des Falles nicht mit berücksichtigt ist die Beobachtung von Hermann ${ }^{3}$ ), der über einen Fall von Perivaginitis berichtet.

Schliesslich sei hier noch zweier Fälle-von Liebermeister ${ }^{4}$ ) Erwähnung gethan. In beiden Fällen war Typhus abdom. vorhergegangen, der eine Fall ging nach anfänglicher Bildung einer Rectovaginalfistel in Genesung über, der andere Fall (74jährige Frau) ging unter Bildung eines Abscesses zwischen Rectum und Vagina mit Durchbruch in das Rectum zu Grunde. Ob ersterer Fall einwandsfrei hierher gehört; will ich nicht entscheiden.

$\mathrm{Zu}$ der Zeit, wo ich einen Fall von Perivaginitis phlegmonosa dissecans zu beobachten Gelegenheit hatte, trat $\mathrm{Busse}^{5}$ ) mit seiner Arbeit an die Oeffentlichkeit. Patientin war wegen Scheidenatresie, hervorgerufen durch lockere Verwachsungen, behandelt worden, indem letztere stumpf gelöst, die Scheide tamponirt und wegen Blutungen mit Liq. ferr. sesquichl. geätzt wurde. Nach einigen Monaten gaben die gleichen Verhältnisse zu demselben Verfahren und dieses Mal zur Tamponade der Scheide mit in Liq. ferr. sesquichl. und Zinc. chlor. getauchten Tampons, die drei Tage liegen blieben; Veranlassung, worauf sich nach einigen 'Tagen das Scheidenrohr in toto als starrwandiger, lederharter, $9 \mathrm{~cm}$. langer Körper mitsammt der Port. vag. ablöste. Im mikroskopischen Schnitt fand man

1) Handbuch d. Gynäkol. Bd. I. S. 318.

2) Annales de Gyn. 1875. p. 235.

3) Jahresber. d. Leistungen u. Fortschritte d. ges. Med. 1887. Bd. 2. p. 712 .

4) Handb. d. spec. Path. u. Therapie v. Ziemssen. Bd. 2.

5) Dieses Archiv. Bd. 56. H. 3. 
eine innere kernlose und eine äussere auf das Dichteste mit Körnern erfüllte, den äusseren Lagen der Muskulatur und dem perivaginalen Gewebe entsprechende Schicht. Die Gefässe waren von obturirenden Thromben erfüllt.

Busse beruft sich auf einen Fall von Nammack ${ }^{1}$ ), der gleichfalls nach dem Gebrauch von Eisenchlorid eine Abstossung der Scheide beobachtete.

Die Reihe der Beobachtungen schliesst mit der jüngst erschienenen Publikation von Bodenstein ${ }^{2}$ ), der zwei Fälle anfübrt. In dem einen derselben hatte sich ohne nachweisbares aetiologisches Moment eine geringe fluctuirende, ohne scharfe Ränder in die teigige Umgebung übergehende Geschwulst gebildet. Der Fall ging unter Ausstossung eines Stückes aus der Scheide und geringer Stenosenbildung in Genesung über. Im zweiten Fall handelte es sich um einen paravaginalen Abscess, der nach Incision zur Heilung führte. Bodenstein glaubt in.seinen zwei Fällen das Anfangsstadium der Perivaginitis phlegmonosa dissecans beobachtet $\mathrm{zu}$ haben, und in der That ist es nicht unmöglich, dass die Perivaginitis im Beginn ihrer Entwickelung dieses Stadium durchläuft, welches bisher der Beobachtung entgangen ist.

Die Anzahl der in der Litteratur beschriebenen Fälle, die ich in ihrer Vollständigkeit glaube erwähnt zu haben, beträgt demnach 17, von denen drei letal endeten (Minkiewitsch, Dobbert, Liebermeister je ein Fall) und 14 in Genesung übergingen, davon laut Angabe 8 Fälle mit nachfolgender Verengerung der Scheide verschiedenen Grades. Als aetiologisches Moment figurirt Typhus abdom. 5 mal, Pneumon. croup. $1 \mathrm{mal}$, fieberhafte catarrhalische Bronchitis $1 \mathrm{mal}$, lokale Behandlung mit Eisenchlorid 2 mal, in 8 Fällen ist die Aetiologie in vollkommenes Dunkel gehüllt und dieses offen ausgesprochen oder den verschiedensten Vermuthungen Raum gegeben.

Bevor wir diese eigenartige Krankheit unter dem aetiologischen Gesichtspunkt betrachten, sei es gestattet, in den folgenden Zeilen der Krankengeschichte meiner Patientin Raum zu geben. -

Akulina Iwanowa, 39 Jahre alt, verheirathet, Wäscherin, hat nicht geboren, menstruirte nicht immer regelmässig. Zwei Monate war die Regel ausgeblieben, darauf litt sie $2 \frac{1}{2}$ Monate an Blutungen, die letzte Zeit nicht reichlich. Sie klagt über Rücken-

1) Schmidt's Jahresber. 1895. Jahrg. XXIX. Bd. II.

2) Monatsschr. f. Geb. u. Gyn. 1898. Bd. VIII. H. 6. 
schmerzen und Schmerzen im Epigastrium und will in der letzten Woche gefiebert haben. Uebelriechenden Ausfluss hat sie nicht bemerkt und nie Ausspülungen der Scheide gemacht.

Am 18. 9. 1898 wurde folgender Status aufenommen:

Kräftig gebaute Frau von gutem Ernährungszustand. Vagina weit. An Stelle der Port. vag. fühlt man einen weichen, schwammigen Körper, ebenso ruft das Tastgefühl, welches der explorirende Finger bei Berührung des Scheidengewölbes, sowie des oberen Theiles des Scheidenrohres erfährt, einen vom normalen abweichenden Eindruck hervor, indem sich diese Partieen ebenfalls gleichsam schwammig-weich anfühlen. Der Uterus ist anteflectirt, beweglich, nicht vergrössert, die Adnexa und Parametrien sind normal.

Nach Einführung des Speculums sieht man, dass die Port. vag. in eine schmutzig graue, weiche gangränöse llasse verwandelt ist, wobei sich die vordere und hintere Muttermundslippe mit dem dazwischenliegenden Muttermund noch unterscheiden lassen. Der gangränöse Zerfall setzt sich ringsum auf das Scheidengewölbe fort und nimmt einen grossen Theil der seitlichen, vorderen und hinteren Scheidenwände, von letzterer $2 / 3$ ihrer Ausdehnnng; ein. Das gesunde Gewebe ist vom gangränösen durch einen scharfen Demarcationsrand getrennt, das gangränöse Gewebe lässt sich unter geringer Blutung von der Unterlage etwas abheben. Schmutzig graue, jauchige, stinkende Absonderung. Die Behandlung bestand in Irrigation der Scbeide und Tamponade mit Jodoformmarly.

Bei der mikroskopischen Untersuchung des Secretes wurden Staphylokokken und Streptokokken einzeln und in Haufen gefunden, ausserdem einige degenerirte Plattenepithelien aus der Scheide.

Die Temperatur blieb die ganze Zeit über normal, die höchste Temp. betrug 37,6 .

Am 20. 9.; also am dritten Tage des Aufenthaltes im Krankenhaus, wurde beim Tamponwechsel ein gangränöses Stück mit fetzigen Rändern von $10 \mathrm{~cm}$ Länge, $4 \mathrm{~cm}$ Breite und $1-11 / 2 \mathrm{~cm}$ Dicke von schmutzig grauer, theils grau-schwarzer Farbe ausgestossen. Es erwies sich, dass dieses Stück einen Abguss aus einem Theil der Scheide mit der Port. vag. darstellte und eine Oefinung entsprechend dem äusseren Muttermund hatte. Die Oberfläche ist von der einen Seite ziemlich glatt, die andere der Scheidenwand zugekehrte Fläche ist uneben, zottig. Nach Abstossung des necrotischen Stückes präsentirt sich die Port. vag. als kleiner hochrother, sammetartiger Zapfen, rund herum hat die Vagina das Aussehen einer frischen Wundfäche, theilweise ist dieselbe noch von einem oberflächlichen Belag bedeckt.

Der weitere Verlauf gestaltete sich in der Weise; dass die entblösste Schleimhaut sich reinigte und mit frischem Granulationsgewebe bedeckte. Die Absonderung war anfangs noch jauchig, später blutig tingirt und hörte alsbald ganz anf.

Als die Kranke am 6. 10, also am 18. Tage ihres Aufenthaltes im Krankenhause auf ihren ejgenen Wunsch entJassen wurde, war die Schleimhaut mit einem frischen Epithelbezug versehen. Der Aufforderung, sich nach einiger Zeit wieder vorzustellen, ist Pat. nicht gefolgt, so dass nir über das weitere Schicksal der Kranken nichts bekannt ist.

Die mikroskopische Untersuchung des : ausgestossenen Stückes er- 
wies, dass dieses aus einem homogenen; structurlosen Gewerbe bestand, welches den Farbstoff schlecht annahm. Stellenweise lassen sich im homogenen Gewebe aufgelockerte fibrillär angeordnete Bindegewebsfasern erkennen. Das Oberflächenepithel ist vollkommen zerstört. Die nicht zahlreichen quer- und längsgetroffenen Gefässlumina sind thrombosirt, die Gefässwände bieten keine auffallende Veränderung dar. Auf dem nach Gram gefärbten Präparat sieht man zahlreiche Colonien von Streptokokken, in Ketten und Haufen angeordnet. Vorzugsweise finden sich dieselben auf der Oberfläche an Stelle der zerstörten Schleimhaut, dann aber auch in der Umgebung der thrombosirten Gefässe und in den Thromben selbst.

Der soeben beschriebene Fall kann als Analogon dem vorher mitgetheilten an die Seite gestellt werden.

Können wir in unserem Fall ein ursächliches Moment ausfindig machen, welches die Ausscheidung eines grossen Theiles der Vagina, des Scheidengewölbes und der Port. vag. zur Folge gehabt hat?

Der Versuch einer Erklärung ist mehrfach angetreten worden, man ist jedoch über gewisse Muthmassungen nicht hinausgekommen. Besonders haben es die älteren Autoren vermieden, die dunkle Seite der Aetiologie zu berühren. Auch unter den jüngeren Autoren ist Busse ${ }^{1}$ ) der einzige, welcher die Frage nach der Entstehung am meisten berücksichtigt und am ausführlichsten erörtert hat. Auf die Ausführungen dieses Autors kommen wir weiter unten zurück.

Man hat vielfach angenommen, dass sich ein entzündlicher Prozess sui generis im lockeren die Scheide umgebenden Zellgewebe gebildet und dieser primäre im paravaginalen Gewebe sich abspielende Entzündungsprozess sich auf die Vaginalwand fortgesetzt habe, so dass diese der Necrose verfalle und wie eine Art Sequester abgestossen werde. So schreibt Breisky ${ }^{2}$ ): Selbstverständlich ist die Obliteration der Scheide nicht ausschliesslich das Ergebniss von Prozessen, die sich auf die Schleimhaut beschränken, sondern spielen die begleitenden Entzündungen im perivaginalen Bindegewebe dabei recht häufig eine wesentliche Rolle."

Doch ist diese Erklärungsweise mit den anatomischen Verhältnissen und der Art und Weise der Lokalisation und der Ausbreitung der Perivaginitis nicht in Einklang zu bringen. Denn das paravaginale Gewebe steht bekanntlich, wie Busse in seiner oben

1) l. c.

2) Handb. d. allgen. u. spec. Chirurgie. Bd. IV. Abthl. 1. S. 58. 
citirten Arbeit ausführt, durch reichlich entwickelte Lymph- und Blntbahnen mit dem Fett- und Zellgewebe des Beckens in naher Communication. und eine sich im paravaginalen Gewebe abspielende phlegmonöse Entzündung musste dem Charakter der Phlegmone entsprechend und den lokalen Verhältnissen gemäss unbedingt in Kürze weit um sich greifen. Statt dessen soll die Perivaginitis ausschliesslich in der unmittelbaren Umgebung der Scheide ihren Sitz haben und beschränkt bleiben, obgleich diese, wie gesagt, von der Nachbarschaft durchaus nicht so streng abgeschlossen ist.

Von Anderen ist die Erscheinung in der Art gedeutet worden, dass die Entzündung ihren Weg von der Scheide aus durch die Wandung derselben genommen hat (Weber). Durch Aufhebung der Integrität der Scheidenschleimhaut sei den Entzündungswegen die Möglichkeit gegeben, in und durch das Gewebe selbst vorzudringen und in der Umgebung der Scheide eine Entzündung hervorzurufen. Doch spricht der Umstand gegen diese Auffassung, dass Wunden auf der Scheidenschleimhaut, sei es operativ angelegte oder anderweitig erworbene, sowie entzündliche Veränderungen der Schleimhaut, zu den alltäglichen Vorkommnissen gehören, ohne dass auch nur ein Fall bekannt wäre, der eine Perivaginitis zur Folge gehabt hätte.

Busse bringt die bisher bekannten Fälle in der Litteratur, die er übrigens nicht in ihrer Vollzähligkeit anführt, in Zusammenhang mit schweren fieberhaften Infectionskrankheiten. Wie auf Schleimhäuten anderer Körpergebiete, wie dem Rachen, Kehlkopf etc. so könne auch hier eine Schädigung der Vagina, sei es durch Ansiedelung der Krankheitserreger selbst an Ort und Stelle, sei es durch die im Blut kreisenden Umsatzstoffe der in anderen Organen angesiedelten Bacterien zu Stande kommen. Hierzu disponire der voraufgegangene Kräfteverfall der Patientinnen. Die Wirkung der Ptomaine bei den Infectionskrankheiten habe, ebenso wie die Wirkung des Eisenchlorids in dem von ihm beobachteten Fall, dieselbe Wirkung, nämlich die Mortification der Scheidenschleimhaut. Die eitrige perivaginale Entzündung sei keineswegs eine Phlegmone im gewöhnlichen Sinne, man thäte vielmehr besser, den Prozess als eine Kolpitis gangraenosa oder necrotica zu bezeichnen.

Ueberblicken wir die oben angeführten Fälle, so fällt uns sofort der Umstand ins Auge, dass in fast jedem Fall das necrotisehe Stück aus Gewebselementen der Scheidenwand und 
604 v. Lingen, Ein Fall von Perivaginitis phlegmonosa disşecans.

der Port yag. besteht. Dieses ist. kein zufälliges Zusammentreffen, wogegen schon an und für sich die in dieser Beziehung auffallende Uebereinstimmung der Fälle sprechen würde, sondern hat auch seinen triftigen Grund in den anatomischen Verhältnissen und basirt auf den elementarsten entwickelungsgeschichtlichen Gesetzen.

Vergleichend - anatomische und embryologische Untersuchungen haben zur Genüge dargethan, dass die Vaginalportion, speziell die vaginale Portion der Cervix (Schroeder), entwickelungsgeschichtlich als ein Bestandtheil der Scheide aufgefasst werden muss. Ich verweise unter Anderem auf die in jüngster Zeit erschienene fleissige und ausführliche Arbeit von Werth und Grusdew ${ }^{1}$, welche die Entwickelung der Morphologie, speziell der Uterusmuskulatur an einer Reihe von foetalen, kindlichen und geschlechtsreifen Uteris zum Gegenstand ihrer Untersuchung machten. Ein Blick auf Fig. 7 der-Tafel XVIII dient zur Erläuterung der uns speziell interessirenden Frage, auf die hier näher einzugehen wir uns versagen müssen.

- Entsprechend der Zugehörigkeit der Port. vag. zur Scheide vom entwickelungsgeschichtlichen Standpunkt ist die Gefässvertheilung auch eine ganz charakteristische. Auf diesem Gebiet hat sich $\mathrm{Nagel}^{2}$ ) durch seine anatomischen Studien mit Freilegung und Injection der Gefässe an Leichen die grössten Verdienste erworben.

Der obere Theil der Scheide und die Vaginalportion verdanken gemeinsam grösstentheils einem Zweige der Art. uterina, nämlich der Art. cervico-vaginalis, ihre Ernährung. "Die Arteria cervico-vaginalis läuft der Seitenkante des Mutterhalses entlang abwärts und löst sich in mehrere Zweige auf, von welchen einige zur vorderen und hinteren Cervixwand gehen, um entweder dort zu endigen oder weiter zur vorderen und hinteren Scheidenwand zu ziehen, während andere direct zur Scheide gehen. Die Cervicalzweige verbinden sich mit deujenigen der Art. uterina der anderen Seite zu ausgebildeten Anastomosen, welche besonders an dem schwangeren Uterus deutlich hervortreten. Einer von ihnen biegt zuweilen in der Mittellinie der hinteren (Hyrtl) oder der vorderen Cervicalwand rechtwinkelig nach unten und geht auf das hintere beziehungsweise vordere Scheidengewölbe über, um in der Mittellinie der Scheidenwand nach abwärts zu verlaufen: die von Hyrtl beschriebene Art. azygos vaginae" (Nagel). Letztere anasto-

1) Dieses Archiv. Bd. 55. H. 2.

2) Dieses Archiv. Bd. 53. H. 3. 
mosirt mit den anderen zur Scheide zichenden Cervicalzweigen, theils mit den Ausläufern der Arteriae vaginales.

Weniger charakteristisch ist das Verhalten der Venen, die mit ihren vielverzweigten Netzen und Geflechten keinen so regelmässigen Befund darbieten, wie die Arterien. Der Plexus vaginalis, der sieh in der Wand der Scheide und des Gebärmutterhalses (Nagel) befindet, bildet eine der Quellen zu der Vena vesico-vaginalis und utero-vaginalis, welche ihrerseits in Bezug auf ihr gegenseitiges Verhalten, Einmündungsstelle, Anastomosen ete. manchen Veränderungen unterworfen sind.

Ebenso streng gesehieden wie die Arterien, ist die Anordnung des Lymphgefässsystems eine der entwickelungsgeschichtlichen Anlage der Scheide und Portio entsprechende. Die Lymphgefässe des oberen Drittels der Scheide sammeln sich; wie Schauta ${ }^{1}$ ) an einer trefflichen Zeichnung illustrirt, in 2-3 Stämme und vereinigen sich mit den vom Collum uteri stammenden Lymphgefässen, um, von der Arteria und Vena uterina begleitet, in transversaler Richtung gegen die seitliche Beckenwand zu verlaufen.

Wir erseben hieraus, dass die Portio vag. und die oberen Partieen der Scheide vom anatomischen Gesichtspunkt aus, sowie in Bezug auf ihre Gefässversorgung in innigè Beziehung zu einander stehen. Sollte nicht dieser Umstand bei gleichmässigem Befallensein von Portio vaginalis und Scheide eine gewisse Rolle spielen?

In der That fanden sich bei einigen Autoren diesbezügliche Andeutungen und Muthmassungen. So wirft Ebermann ${ }^{2}$ ) in der Discussion im Anschluss an den Vortrag Tschernischew's die Frage auf, ob nicht der Befund an den Gefässen zur Klärung der räthselhaften Krankheit beitragen könne, ob es sich nicht um locale Embolien handelt. Die Frage fand bei der Discussion keine Beantwortung. Ebenso streifen Chanutin und Minkiewitsch ${ }^{3}$ ) die aetiologisehe Frage von dieser Seite; Chanutin beantwortet dieselbe aber in negativem Sinne, gleichzeitig auf eine befriedigende Lösung der Frage verzichtend. Minkiewitsch giebt der Vermutbung Raum, dass Thrombose oder Embolie gewisser Gefässe ein aetiologisches Moment abzugeben im Stande wäre.

) Lehrb. d. ges. Gynäk. 1895.

y) Medizinsky Westn. 1880. No. 49 . p. 389 u. 390.

3) 1. c. 
Endlich sprach sich Dobbert ${ }^{1}$ ) bei Gelegenheit meines Vortrages über obiges Thema dahin aus, dass der ganze Prozess in einer Circulationsstörung der betreffenden Theile seine Ursache habe - eine Ansicht, der ich mich anschliesse, nachdem ich mich überzeugt hatte, dass anderweitige aetiologische Gründe, nach welchen zu forschen ich mich veranlasst fühlte, weit weniger stichhaltiger Natur seien.

Den Vorgang kann man sich in der Weise vorstellen, dass eine Verstopfung gewisser Gefässgebiete, sei es embolischer, sei es thrombotischer Genese, zu Stande kommt und die Ausschaltung eines Gefässgebietes, eine Schädigung des von den betreffenden Gefässen versorgten Gewebsterritoriums und eine Einbusse desselben in seiner Ernährung und Lebensfunction zur Folge hat, die schliesslich zur Nekrose führen muss. Der Vorgang ist ein ähnlicher, wie derselbe von $\mathrm{P}$ op off ${ }^{2}$ ) beim hämorrhagischen Infarkt des Uterus geschildert, und gehen wir einen Schritt weiter, wie derselbe bei der Gangraena senilis oder dem Decubitus beobachtet wird.

Das seltene Vorkommen des hämorrhagischen Infarktes des Uterus sieht Popoff mit Recht in den ungemein günstigen Bedingungen der Blutcirculation im Uterus, indem dieser das Blut aus zwei bilateralen Quellen (Arteriae uterinae und Arteriae spermaticae) erhält.

$\mathrm{Ob}$ und welche Veränderungen an den Gefässen der Genitalorgane eine Disposition zur embolischen oder thrombotischen Verstopfung der Gefässe bilden, entzieht sich vorläufig dem Nachweise, da die meisten Patientinnen mit Perivaginitis phlegmonosa dissecans geheilt entlassen werden, an den Verstorbenen aber eine mikroskopische Untersuchung der Gefässe nicht ausgeführt worden ist. Popoff fand bei einer an ehronischer Endocarditis und Nephritis gestorbenen 40 jährigen Patientin neben einem Lungeninfarkt auch einen solchen der Cervix uteri. Die Aeste der Arteria uterina wiesen verschiedene pathologische Veränderungen auf, wie Kalkinfiltration, Endarteriitis chronica bis zur vollständigen Obliteration der Gefässe, frische und alte Thromben.

1). Protokolle der Sitzungen d. Peter-Paul-Krankenhauses zu St. Petersburg. 1899. (Russisch.)

2) Dieses Archiv. Bd. 47. H. 1. 
Bereits unter normalen Verhältnissen ist die Struetur der Arteria uterina nach den Untersuchungen Westphalen's ') in hohem Grade von den übrigen peripheren Körperarterien verschieden und nach stattgehabter. Geburt gehen die Uteringefäss $\theta$ eine Reihe von Veränderungen ein $\left(\right.$ Balin) ${ }^{2}$ ).

Herxheimer ${ }^{3}$ ) beschreibt den Sectionsbefund einer an einer chronisch-hämorrhagischen Nephritis Gestorbenen, bei dem sich neben Hypertrophie des Herzens, Lungeninfarkten etc. auch eine „Infarcirung mit Gangrän der Scheide, der Portio vagin., des unteren Theiles des Rectums, der Blase und des Perineums, Gangrän im Douglas'schen Raum und Peritonitis" fand. Die rechte Art. hypogastrica war an ihrer Ursprungsstelle durch einen reitenden Embolus verstopft, linkerseits waren die Arteriae uterina und vaginalis, die Arteriae haemorrhoidales externae und die Art. haemorrh. media, sowie die Art. perinealis embolisirt. Dadurch war eine Ernährungsstörung, wie sie zum Eintritt eines hämorrhagischen Infarktes nöthig ist, entstanden. „Dass dẹ hämorrhagische Infarkt hier nicht nur einen Gewebstod, sondern einen solchen plus Fäulniss, d. h. Gangrän erzeugte, ist bei der für Fäulnisskeime so exponirten Lage der Organe nicht wunderbar. ${ }^{*}$

Bei der Perivaginitis phlegmonosa dissecans des oberen Theiles der Scheide musste es sich entsprechend den anatomischen Verhältnissen um eine Verstopfung der die Port. vag. und die oberen Partien der Scheide versorgenden Gefässe handeln. Entsprechend der circulären Anordnung der Gefässe um Port. vag. und Vagina verfällt demnach auch ein circulärer Gewebsantheil - nämlich die ebengenannten - der Nekrose.

"Nit dieser Auffassung liesse sich, wie mir schent, auf anatomischer Grundlage basirend, am ungezwungensten die Uebereinstimmung erklären, mit der jedes Mal von den Autoren notirt ist, dass Portio und ein mehr weniger grosser Antheil des Scheidenrohres ausgestossen wird. Ich stehe nicht an, zu behaupten, dass dieser Erklärungsmodus auf alle Fälle anzuwenden ist, dagegen hat derselbe für einzelne Fülle, wenn nicht für die Mehrzahl, wie aus den vorhergehenden Auseinandersetzungen hervorgeht, Manches für sich. Die Fälle von Busse und Nammack., in denen Portio

1) Virch. Arch. Bd. 106. H. 2.

2) Dieses Archiv. Bd. 15. H. 2.

3) Virchow's Archiv. Bd. 104. H. 1. 
und Scheide nach localer Anwendung von Eisenchlorid eliminirt wurden, würden allerdings auf diese Weise nicht erklärt werden können. Hier liegt eine locale Aetzwirkung der Vaginalschleimhaut und der tieferen Schichten durch Eisenchlorid und Chlorzink vor - ein Vorgang, wie Bodenstein richtig bemerkt, der mit dem Process der Perivaginitis nur den Enderfolg gemeinsam hat. Als ursächliches Moment für derartige Circulationsstörung können acute Infectionskrankheiten (Typhus abdom., Pneumonie etc:) oder anderweitige fieberhafte Erkrankungen angeführt werden, indem unter dem Einfluss herabgesetzter Allgemeinernährung im Stadium decrementi auch die Blutcirculation an Energie Einbusse erleiden und wie in anderen Organen (Gehirn, Milz etc.) auch gelegentlich ein $\mathrm{Mal}$ in den Aesten der Art. uterina eine Embolie hervorrufen kann. Oder aber es kommt in Folge eines localen entzündlichen Processes in der Umgebung der Vagina zur Thrombose des benachbarten Gefässes, wodurch dann ein grösserer Bezirk ausser Function gesetzt der Nekrose anheim fällt. In unserem Fall war gleichfalls dem Eintritt in das Krankenhaus eine acut fieberhafte Erkrankung unbekannter Natur vorhergegangen. Die Anwesenheit der Streptokokken in dem eliminirten Gewebsstück, und zwar vorzugsweise auf der Oberfläche der pathologisch veränderten Schleimhaut, den Thromben in den Gefässen und in der Umgebung derselben deutet darauf hin, dass die Mikroorganismen den Thrombus durchsetzt, von diesem aus das Gewebe durchdrungen and in dem in seiner Vitalität herabgesetzten Gewebsbezirk den nöthigen Nährboden gefunden haben.

Was den klinischen Verlauf der Perivaginitis betrifft, so bieten die objectiven Anzeichen wenig Charakteristisches: bei mehr weniger ausgesprochenen Fiebererscheinungen besteht stärkerer übelriechender Ausfluss und Schmerzen im Unterleib. Der missfarbene nekrotische Bezirk wird unter den Erscheinungen der Demarcation ausgestossen, worauf sich die entblösste Wundfläche mit frischen Granulationen bedeckt und unter mehr weniger ausgesprochener Narbenbildung der Heilung entgegengeht.

Die Prognose ist, wie aus dem Vorhergehenden hervorgeht, meist günstig zu stellen.

Die Behandlung beschränkt sich auf desinficirende Ausspülungen und späterhin Tamponade der frisch granulirenden Wundfläche, um nach Möglichkeit einer vorzeitigen Verklebung der Wundfï̈chen und der Bildung von Atresien entgegenzuwirken. 